\title{
Postoperative Endophthalmitis Caused by Candida Parapsilosis: A Case Report
}

\author{
María del Pilar Lucena ${ }^{1}$, Federico Furno Sola ${ }^{1}$, Mitzy E. Torres Soriano², \\ Fanny Andrea Gerster ${ }^{1}$ \\ ${ }^{1}$ Ophthalmology Department, Sanatorio Mapaci, Grupo Laser Visión, Rosario, Argentina \\ ${ }^{2}$ Retina Department at Centro de la Visión Gordon-Manavella and Clínica de Ojos “Dr. Carlos Ferroni”, Rosario, Argentina \\ Email:pilarlucena@gmail.com,furnosola@gmail.com, mitzytorres@yahoo.com, fannygerster@gmail.com
}

How to cite this paper: Lucena, M. del P., Sola, F.F., Soriano, M.E.T. and Gerster, F.A. (2019) Postoperative Endophthalmitis Caused by Candida Parapsilosis: A Case Report. Open Journal of Ophthalmology, 9, 64-69. https://doi.org/10.4236/ojoph.2019.92008

Received: November 27, 2018

Accepted: April 12, 2019

Published: April 15, 2019

Copyright $\odot 2019$ by author(s) and Scientific Research Publishing Inc. This work is licensed under the Creative Commons Attribution International License (CC BY 4.0).

http://creativecommons.org/licenses/by/4.0/

\begin{abstract}
Purpose: To report an unusual case of postcataract endophthalmitis secondary to Candida parapsilosis. Case Presentation: A 77-year-old female patient was admitted to the emergency ward with reduced visual acuity (VA), and redness and pain in the right eye (RE) with a few hours of evolution. The patient underwent cataract surgery in the right eye 50 days earlier; no abnormalities were discovered in postsurgery check-ups on the following days. Biomicroscopy revealed corneal oedema, tyndall ++++ , and flare +++ . Capsule-shaped whitish deposits and posterior synechiae were also reported. Fundoscopy: not visible due to anterior segment opacity. RE ultrasound: mild vitritis, attached retina. Due to a strong suspicion of endophthalmitis, an aqueous humour biopsy was performed and the presence of Candida parapsilosis sensitive to amphotericin B and voriconazole was revealed. In view of a poor prognosis, we decided to carry out a vitrectomy, removal of the IOL + capsular bag complex and intravitreal injection of amphotericin B. After several check-ups, the patient reported being diagnosed with a fungal infection in her fingernails some time ago. She was referred for a microbiological testing, which confirmed the presence of Candida in the fingernails. Conclusions: Fungal endophthalmitis is uncommon in the postoperative setting, with most of the cases being attributable to the Candida species. The combination of pars plana vitrectomy, removal of the IOL + capsular bag complex and antifungal agents was able to successfully treat this case of postoperative endophthalmitis due to Candida parapsilosis. The relationship between postoperative Candida endophthalmitis and Candida nail infection is dramatic. To date, only one case of endogenous endophthalmitis associated with complicated onychomycosis has been reported.
\end{abstract}




\section{Keywords}

Endophthalmitis, Candida, Cataract Surgery, Nail Infection

\section{Introduction}

One of the most devastating complications after ocular surgery is endophthalmitis. Cataract surgery represents the majority of ocular surgery procedures; thus, most of the reported cases of endophthalmitis in the literature were after cataract surgery. The rate of endophthalmitis varies from $0.04 \%-0.2 \%$ [1].

Chronic postoperative endophthalmitis (CPE) is a delayed infectious intraocular inflammation process that occurs more than six weeks after ocular surgery. In explanted intraocular lenses (IOL), histopathological examination has revealed the presence of bacteria in relation to the lens capsule and/or on the IOL surface, with intracapsular plaques and inflammatory cells such as lymphocytes, plasma cells and neutrophils. Less commonly, fungi (especially Candida spp.) may be identified [1].

Visual outcomes are not favourable. Some studies report that approximately $40 \%$ of affected patients experience severe visual loss (BCVA $<20 / 200$ ), and only one-third of cases reach visual acuity of better than 20/40 [1].

Approaches to treatment often include antifungal intravitreal injection and/or surgery including pars plana vitrectomy (PPV), intraocular lens removal, and total capsulectomy.

Candida parapsilosis is rare but can have devastating effects on vision. It is one of the atypical causes of endophthalmitis after cataract surgery [1].

We report in this article a case of a female patient with pseudophakic Candida parapsilosis endophthalmitis and onychomycosis caused by Candida.

\section{Case Report}

A 77-year-old female patient was admitted to the emergency ward with reduced visual acuity (VA) and redness and pain in the right eye (RE) with a few hours of evolution. Medical history: high blood pressure treated with atenolol 20 years earlier. Ocular history: cataract surgery in the left eye (LE) 3 months earlier. Cataract surgery in the right eye 50 days earlier; no abnormalities were discovered in postsurgery check-ups on the following days.

Best-corrected visual acuity (BCVA): hand motion. Biomicroscopy revealed corneal oedema, tyndall ++++ , and flare +++ . Capsule-shaped whitish deposits and posterior synechiae were also observed (Figure 1). Intraocular pressure (IOP): $9 \mathrm{mmHg}$. Fundoscopy: not visible due to anterior segment opacity. RE ultrasound: mild vitritis, attached retina. Treatment indicated: prednisolone + phenylephrine every 3 hours, cyclopentolate every 12 hours and moxifloxacin every 3 hours. 


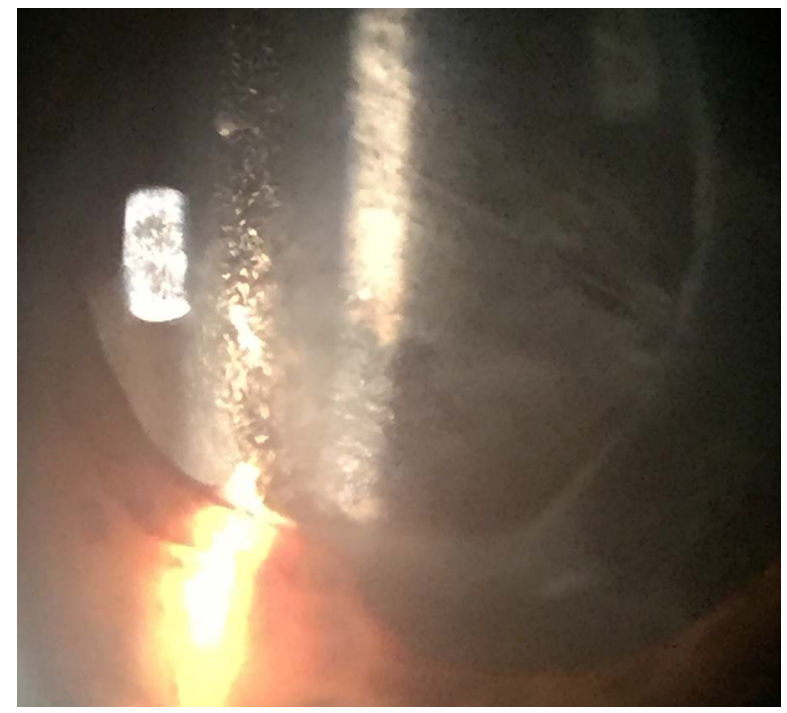

Figure 1. Slit-lamp biomicroscopy images of the right eye showing signs of inflammation.

Due to a strong suspicion of endophthalmitis, an aqueous humour biopsy and empiric treatment with intravitreal injection of vancomycin and ceftazidime were performed.

The direct analysis of the aqueous humour revealed the presence of yeasts. Amphotericin B $(0.005 \mathrm{mg} / 0.1 \mathrm{ml})$ was administered through intravitreal injection and oral voriconazole treatment was started (200 mg every 12 hours). Aqueous humour culture revealed the presence of Candida parapsilosis sensitive to amphotericin B and voriconazole.

In view of a poor prognosis, we decided to carry out a vitrectomy, removal of the IOL + capsular bag complex and intravitreal injection of amphotericin B. Microbiological testing of the biopsy specimen revealed the presence of Candida parapsilosis in the capsular bag.

On the following day, during the postsurgery check-up, the patient's RE BCVA was $1 / 10$ with a positive lens of $12.00 \mathrm{D}$. Biomicroscopy exam showed aphakia, tyndall ++++, and flare +++. Fundoscopy revealed an attached retina.

The patient progressed favourably after vitrectomy. Four days after surgery, another injection of amphotericin B was administered.

Twelve days after surgery, the patient reported pain once again and biomicroscopy showed retrocorneal precipitates and tyndall +++ . The amphotericin $B$ injection was repeated, and topical prednisolone was administered every 2 hours was indicated. After 2 weeks, the retrocorneal precipitates and inflammation had disappeared.

Weekly amphotericin B injections were administered for 2 weeks.

Postoperative check-up (2-month progress): BCVA was 6/10 and IOP was 10 $\mathrm{mmHg}$. Biomicroscopy showed a clear cornea, aphakia, no reactions in the anterior chamber, and no retrocorneal precipitates. Fundoscopy revealed an attached retina. 
After several check-ups, the patient reported being diagnosed with a fungal infection in her fingernails some time ago. She was referred for microbiological testing, which confirmed the presence of Candida in the fingernails.

Four-month follow-up after surgery (Figure 2): BCVA was 9/10 and IOP was $10 \mathrm{mmHg}$. Biomicroscopy: clear cornea, aphakia, no reactions in the anterior chamber, and no retrocorneal precipitates. Fundoscopy revealed an attached retina.

At present (9 months after the surgery), the patient remains aphakic, without signs of inflammation, and is not undergoing antifungal or steroid treatments; the aqueous humour cultures are negative for fungi. Her BCVA is $9 / 10$, and she is waiting for a secondary IOL implant.

\section{Discussion}

C. parapsilosis is a rare cause of chronic pseudophakic endophthalmitis that can have devastating effects on vision.

In this case, the diagnosis of postoperative late-onset fungal endophthalmitis was based on the patient's clinical signs and the results of the aqueous humour and vitreous cultures.

We used intravitreal injections of amphotericin B because of its efficiency against a wide range of fungal pathogens [2]. The recommended dosage range is $5 \mathrm{mcg}$ to $10 \mathrm{mcg}$. Retinal necrosis and retinal detachment have been reported with doses greater than $25 \mathrm{mcg}$ injected very close to the retina [3].

There is no consensus about the surgical management of fungal endophthalmitis. We decided to perform an extensive vitrectomy associated with IOL-capsular bag removal to eradicate the fungus in the intraocular dead-spaces where antifungal therapy cannot diffuse [4].

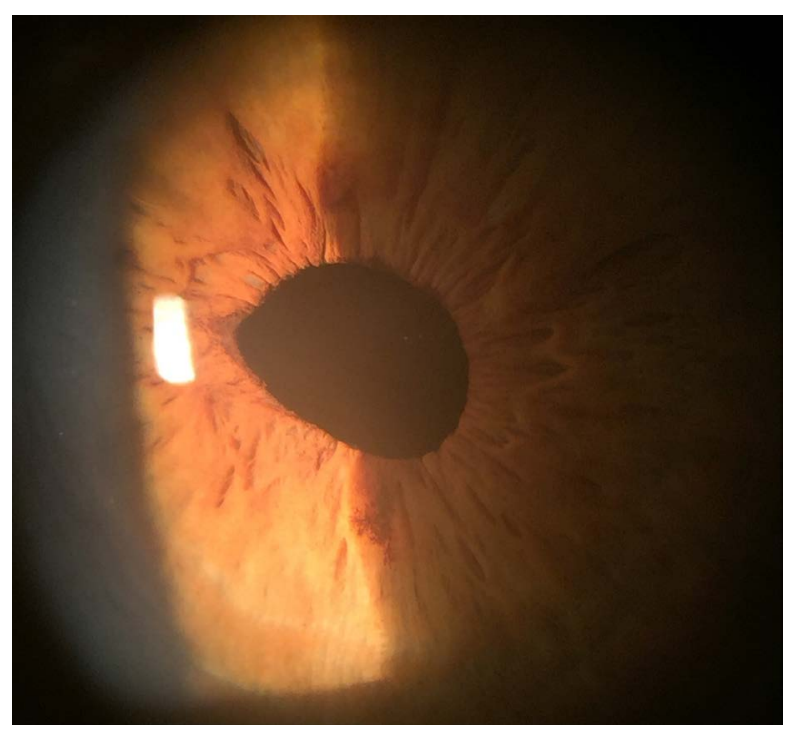

Figure 2. Four-month follow up after surgery. Slit-lamp biomicroscopy picture of the right eye shows aphakia without signs of inflammation. 
There are several cases published describing cases of endophthalmitis by Candida after cataract surgery. It has also been described the infection by Candida parapsilosis in keratitis after corneal transplants or Post LASIK [5] [6].

The association between the postoperative endophthalmitis and onychomycosis caused by the same fungi is truly interesting; nevertheless, we cannot confirm that the endophthalmitis was due to the nail infection.

\section{Conclusions}

Postoperative endophthalmitis is a common complication of any intraocular surgery.

The incidence of CPE is very low. Multiple pathogens have been reported. Fungal endophthalmitis is uncommon in the postoperative setting, with most of the cases due to the Candida species.

The combination of pars plana vitrectomy, removal of the IOL + capsular bag complex and antifungal agents was able to successfully treat this patient who had postoperative endophthalmitis due to Candida parapsilosis [7].

In our case, the relationship between postoperative Candida endophthalmitis and Candida nail infection is dramatic. To date, only one case of endogenous endophthalmitis associated with complicated onychomycosis has been reported [8].

\section{Acknowledgements}

The authors acknowledge Dr. D'Alessandro for the help in the diagnosis and treatment of the patient.

\section{Conflicts of Interest}

The authors declare no conflicts of interest regarding the publication of this paper.

\section{References}

[1] Jabarvand, M., Hashemian, H., Khodaparast, M., Jouhari, M., Tabatabaei, A. and Rezaei, S. (2016) Endophthalmitis Occurring after Cataract Surgery: Outcomes of More Than 480,000 Cataract Surgeries, Epidemiologic Features, and Risk Factors. Ophthalmology, 123, 295-301. https://doi.org/10.1016/j.ophtha.2015.08.023

[2] Friling, E., Lundstrom, M., Stenevi, U. and Montan, P. (2013) Six-Year Incidence of Endophthalmitis after Cataract Surgery: Swedish National Study. Journal of Cataract \& Refractive Surgery, 39, 15-21. https://doi.org/10.1016/j.jcrs.2012.10.037

[3] Packer, M., Chang, D.F., Dewey, S.H., et al. (2011) Prevention, Diagnosis, and Management of Acute Postoperative Bacterial Endophthalmitis. Journal of Cataract \& Refractive Surgery, 37, 1699-1714. https://doi.org/10.1016/j.jcrs.2011.06.018

[4] Wykoff, C.C., Flynn, H.W., Miller, D., Scott, I.U. and Alfonso, E.C. (2008) Exogenous Fungal Endophthalmitis: Microbiology and Clinical Outcomes. Ophthalmology, 115, 1501-1507. https://doi.org/10.1016/j.ophtha.2008.02.027

[5] Rhem, M.N., Wilhelmus, K.R. and Font, R.L. (1996) Infectious Crystalline Keratopathy Caused by Candida parapsilosis. Cornea, 15, 543-545. 
https://doi.org/10.1097/00003226-199609000-00017

[6] Chen, W.-L., Tsai, Y.-Y., Lin, J.-M. and Chiang, C.-C. (2009) Unilateral Candida parapsilosis Interface Keratitis after Laser In Situ Keratomileusis-Case Report and Review of the Literature. Cornea, 28, 105-107.

https://doi.org/10.1097/ICO.0b013e318184e69b

[7] Yang, Y.S. (2009) Results of Extensive Surgical Treatment of Seven Consecutive Cases of Postoperative Fungal Endophthalmitis. Korean Journal of Ophthalmology, 23, 159-163. https://doi.org/10.3341/kjo.2009.23.3.159

[8] Tamez-Peña, A., Luis, A.G.-G. and Gilberto, L.-J. (2010) Endoftalmitis endógena por Fusarium spp. en un paciente con onicomicosis: reporte de un caso. Revista Mexicana de Oftalmología, 84, 122-126. 